\title{
Common eye diseases in children of rural community in Goro district, Central Ethiopia
}

\author{
Mohammed Shaffi, Abebe Bejiga
}

\begin{abstract}
Background: Very few reports exist regarding the causes of ocular morbidity in children of rural community in Ethiopia.

Objective: To identify the causes of ocular morbidity in children of rural community.

Method: A community-based cross-sectional study was conducted between January 2003 and February 2003 to identify common eye diseases in children. A cluster sample of village were randomly taken from 7-peasant association in Goro district, Southwest Ethiopia. All children ( $<15$ years) in the selected villages were registered and their eyes examined. Visual acuity was also taken for children older than 5 years.

Result: The study included 826 children, 438 (53\%) males and 388 (47\%) females, whose age range from birth to 15 years. One or more ocular disease was detected in 55\% (CI: 52,58) of children examined. Trachoma was found to be the leading cause of ocular morbidity $33.7 \%$ (CI: 32.2, 35.2) followed by refractive error 6.3\% (CI: 6.0, 6.6) and nontrachomatous conjunctivitis 5.9\% (CI: 5.5, 6.3). Refractive error was the leading cause of subnormal and low vision among children aged 6 to 15 years.

Conclusion: Provision of health education and primary eye care as well as the implementation of the SAFE strategy by trained local health personnel will prevent and alleviate the common causes of ocular morbidity in children of this community. [Ethiop.J.Health Dev. 2005;19(2):148-152]
\end{abstract}

\section{Introduction}

It has been estimated that $5 \%$ of blindness worldwide is childhood blindness, though the prevalence varies from region to region. In general, the magnitude of blindness in children is 3 to 4 times greater in developing countries than in the developed nations. According to WHO, there are 1.5 million blind children in the world of which 70 $90 \%$ are in the poorest countries of Africa and Asia. About half a million become blind each year, of whom probably more than half die in childhood. The prevalence of low vision is probably three to four times higher than that of blindness, with about 5 million children being affected worldwide (1).

Children less than 15 years of age make up about $45 \%$ of the population of Ethiopia (2). Ethiopia, as one of the poorest countries with low socioeconomic level and under developed infrastructures suffers from innumerable treatable and preventable disease. Health service coverage is $50.4 \%$ and a significant number of the rural population has no access to any form of modern health care (3). Immunization coverage in this country is amongst the lowest in the world. According to Ethiopian demographic and health study (EDHS) 2000, only 14\% of children aged 12-23 months are fully immunized against the six vaccine preventable diseases including measles (4).

Previous study done in the same region has shown that $51.6 \%$ of children under 10 years of age suffer from active trachoma (5). Screening for ocular abnormalities and low vision in school children of Butajira town revealed one or more ocular abnormalities in $62 \%$ of the students (6). Refractive error was also found to be the leading cause of low vision in this study. There are few sources of population-based data on the prevalence of ocular morbidity and low vision in children. The purpose of this study was to conduct population based survey in Goro District to identify the common causes of ocular morbidity and subnormal vision in children of rural community.

\section{Method}

A community based cross-sectional study was conducted in Goro district between January $1^{\text {st }} 2003$ and February $28^{\text {th }}$ 2003. Goro district is located in Guragie zone of southern region, 155 kilometers West of Addis Ababa, the capital city of Ethiopia. There were 64 peasant associations (PA) in the district. There was one health center in the town of the district (wolkite) with one trained ophthalmic nurse. Ethical clearance was obtained from the department of ophthalmology, faculty of medicine of Addis Ababa University. The sample size was calculated using the formula $n=(s / d)^{2} p(1-p)$ where $\mathrm{s}=1.96, \mathrm{~d}=$ precision at $5 \%$ and proportion of $62 \%$ obtained from previous school screening (6). The calculated sample size with an anticipated $90 \%$ response rate was found to be 404 .

Seven PAs were randomly selected by lottery method from the total list of 64 . Then the selected PAs were further divided into villages and a cluster sample of one village was randomly taken from each of the selected PAs. All children whose age were 15 years or less living in these villages were registered by enumerators. Then, these children were examined at their respective villages 
after verbal consent was obtained from the parents. Visual acuity was assessed in each eye, in bright day light, using snellen's "E" chart at 6 meters for children aged 6-15 years. Visual acuity of these children was checked by pinhole if it was less than $6 / 9$ in one or both eyes. The WHO classification of visual impairment was used (7).

Examination of the external eye and anterior segment was made by a senior resident using a pen torch and a $2.5 \mathrm{x}$ magnifying loupe. Posterior segment evaluation was done with direct ophthalmoscope. Then corneal light reflex test and cover-uncover tests were done to evaluate ocular alignment. The possible cause of ocular morbidity and subnormal vision were documented. Children with active trachoma, non-trachomatous conjunctivitis and Bitot's spot were given treatments. Those children requiring further evaluation and treatment were referred to the appropriate eye centers.

Children with active trachoma were given two tubes of tetracycline hydrochloride eye ointment to be applied twice daily continuously for six weeks. Those patients diagnosed as having non-trachomatous conjunctivitis were treated with chloramphenicol eye drop five times daily in the affected eye for seven days. Cases with Bitot's spot were managed using the WHO recommendations, i.e. vitamin A 200,000 IU was given orally on the first, second and seventh days for children older than 1 year of age. Half of the above dose was given for children aged one year or less for three days following the same schedule.
In this study, subnormal vision was taken as the presenting visual acuity (VA) of less than 6/9 in the better eye. The WHO clinical staging for trachoma and xerophthalmia was used (8). Refractive error was considered when subnormal vision improved with a pinhole test. Amblyopia was also considered in a child with subnormal vision in the absence of external eye, anterior segment and posterior segment pathology and the presence of conditions giving rise to it like strabismus and anisometropia.

Data analysis was made by computer program "stata". Proportions were used to present the results. P-value and confidence intervals were also used to indicate level of significance of the findings.

\section{Result}

A total of 826 children participated in the survey and underwent eye examination. There were 438 (53\%) males and $388(47 \%)$ females making the male to female ration of $1.1: 1$. The age range from birth to 15 years, the average being 5.8 years. There were 434 (52.5\%), 310 $(37.5 \%)$ and $82(9.9 \%)$ children in the age group $0-5,6-$ 10 and $11-15$ years respectively. Of them, 55\% (95 CI: $52,58)$ children had at least one ocular abnormality. The major causes of ocular morbidity in this survey were active trachoma (TI\&TF), error of refraction and nontrachomatous conjunctivitis in $33.7 \%, 6.3 \%$ and $5.9 \%$ respectively (Table 1). Clinical xerophthalmia as manifested by Bitot's spot was found in $14(1.7 \%)$ of the children. All cases of xerophthalmia were below the age of 6 years and also were found in only two of the seven villages visited.

Table 1: Causes of ocular morbidity identified among children surveyed

Cause of morbidity $\quad$ Frequency by age (years)

Total (\%)

0-5

6-10

$11-15$

\begin{tabular}{lrrrr}
\hline Active trachoma & $181(22 \%)$ & $91(11 \%)$ & $6(0.7 \%)$ & $278(33.7 \%)$ \\
Refractive error & - & $41(5.0 \%)$ & $11(1.3 \%)$ & $52(6.3 \%)$ \\
NTC* & $37(4.5 \%)$ & $12(1.5 \%)$ & - & $49(5.9 \%)$ \\
Bitot's spot & $14(1.7 \%)$ & - & - & $14(1.7 \%)$ \\
VKC** & $7(0.9 \%)$ & $6(0.7 \%)$ & - & $13(1.6 \%)$ \\
Trachomatous scar & & $2(0.2 \%)$ & $6(0.7 \%)$ & $8(1 \%)$ \\
Strabismus & $2(0.2 \%)$ & $4(0.5 \%)$ & $1(0.1 \%)$ & $7(0.8 \%)$ \\
Trachomatous trichiasis & & $1(0.1 \%)$ & $1(0.1 \%)$ & $2(0.2 \%)$ \\
Miscellaneous & $5(0.6 \% 0$ & $18(2.2 \%)$ & $8(1.0 \%)$ & $31(3.8 \%)$ \\
\hline Total & $\mathbf{2 4 6 ( 2 9 . 8 \% )}$ & $\mathbf{1 7 5 ( 2 1 . 2 \% )}$ & $\mathbf{3 3 ( 4 . 0 \% )}$ & $\mathbf{4 5 4 ( 5 5 \% )}$ \\
\hline *non-trachomatous conjunctivitis & & &
\end{tabular}

A total of 288 children were found to have trachoma of which $96.5 \%$ had active trachoma (IT\&/or TI) while only 10/288 (3.5\%) had inactive trachoma. As seen in Table 2 the prevalence of active trachoma decreases with advancing age with $22 \%, 11 \%$ and $0.7 \%$ children falling in the age group of $0-5,6-10,11-15$ years respectively.
On the other hand, though it is less significant in children; the magnitude of inactive trachoma rises with age. When the sex distribution of active trachoma was considered, $51.8 \%$ was in males and $48.2 \%$ in females. The difference was not statistically significant $(\mathrm{p}=0.09)$. 
Table 2: Distribution of active and inactive trachoma by age among children

\begin{tabular}{|c|c|c|c|c|c|}
\hline \multirow[b]{2}{*}{ Stage of trachoma } & \multicolumn{3}{|c|}{ Frequency by age (years) } & \multirow[t]{2}{*}{ Total (\%) } & \multirow{2}{*}{$\begin{array}{l}\text { WHO cut off } \\
\text { point for } \\
\text { public } \\
\text { significance }\end{array}$} \\
\hline & $\begin{array}{l}0-5 \\
(n=434)\end{array}$ & $\begin{array}{l}6-10 \\
(n=310)\end{array}$ & $\begin{array}{l}11-15 \\
(n=82)\end{array}$ & & \\
\hline Trachomatous follicles (TF) & $112(13.6 \%)$ & $58(7.0 \%)$ & $5(0.6 \%)$ & $175(21.2 \%)$ & $>20 \%$ \\
\hline Trachomatous intense inflammation $(\mathrm{TI})$ & $69(8.4 \%)$ & $33(4.0 \%)$ & $1(0.1 \%)$ & $103(12.5 \%)$ & $>5 \%$ \\
\hline Trachomous scar(TS) & - & $2(0.2 \%)$ & $6(0.7 \%)$ & $8(1.0 \%)$ & \\
\hline Trachomatous trichiasis (TT) & - & $1(0.1 \%)$ & $1(0.1 \%)$ & $2(0.2 \%)$ & $>1 \%$ \\
\hline Trachomatous corneal scar (CO) & - & - & - & - & \\
\hline Total & $181(21.9 \%)$ & $94(11.4 \%)$ & $13(1.6 \%)$ & $288(34.9 \%)$ & \\
\hline
\end{tabular}

Refractive error, corneal scar and amblyopia were the leading causes of subnormal vision as seen in Table 3. While unilateral low vision was seen in $25 / 826(3 \%)$ children, bilateral low vision occurred in $18 / 826(2.2 \%)$ cases. Error of refraction was the cause of unilateral low vision in $16 / 25(64 \%)$ and bilateral low vision in $13 / 18$ $(72.2 \%)$ of the children. No child was found to be blind according to WHO definition. However, unilateral blindness was found in $8 / 826(1 \%)$ of children. Of these, corneal scar contributed to $5 / 8(62.5 \%)$ of the cases.

Table 3: Causes of bilateral subnormal, low vision and unilateral blindness among children aged between 6-15 years old

\begin{tabular}{|c|c|c|c|c|}
\hline Causes & $\begin{array}{c}\text { Subnormal vision } \\
(\%)(V A<6 / 9)\end{array}$ & $\begin{array}{c}\text { Visual impairment } \\
(3 / 60<V A<6 / 18)\end{array}$ & $\begin{array}{l}\text { Unilateral blindness } \\
\text { (VA } \leq 3 / 60)\end{array}$ & $\begin{array}{c}\text { \% Total cases } \\
(n=826)\end{array}$ \\
\hline Refractive error & $39(62.9 \%)$ & $13(72.2 \%)$ & - & $52(6.3 \%)$ \\
\hline Corneal scar & $9(14.5 \%)$ & $1(5.6 \%)$ & $5(62.5 \%)$ & $15(1.8 \%)$ \\
\hline Amblyopia & $10(16.1 \%)$ & $3(16.7 \%)$ & $1(12.5 \%)$ & $14(1.7 \%)$ \\
\hline Nystagmus & $4(6.5 \%)$ & $1(5.6 \%)$ & - & $5(0.6 \%)$ \\
\hline Retinal scar & - & - & $1(12.5 \%)$ & $1(0.1 \%)$ \\
\hline Phthisis bulbi & - & - & $1(12.5 \%)$ & $1(0.1 \%)$ \\
\hline Total & $62(100 \%)$ & $18(100 \%)$ & $8(100 \%)$ & $88(10.7 \%)$ \\
\hline
\end{tabular}

\section{Discussion}

Trachoma has been found to be the major cause of ocular morbidity in this survey. The prevalence of trachomatous follicles $(20.6 \%)$ and trachomatous intense inflammation $(12.4 \%)$ of more than $20 \%$ and $5 \%$ respectively in children of ten years old or less indicates that trachoma is a significant public health disease in Goro District. Though, the overall prevalence of active trachoma (TF \& $\mathrm{TI}$ ) of $33 \%$ obtained among under ten years old children in this study is of public health significance, it has been found to be lower than the previous reports from central Ethiopia (5). Inactive trachoma which includes trachomatous scar and trichiasis was found to be less significant while corneal opacity was even absent. While $96.5 \%$ of the cases had active trachoma, inactive trachoma was found in only 10/288 $(3.5 \%)$ children. This is an expected finding as children are the reservoirs of active disease and its sequelae appears later among adults. The distribution of active trachoma among the sexes was not statistically different too. This is in agreement with previous findings from studies done elsewhere (9).

Non-trachomatous conjunctivitis was the third cause of ocular morbidity in children. This, in addition to active trachomatous infection, is an indication of poor personal and environmental hygienic practice of the community with an over-crowded living condition. This has been noted previously that while children with clean faces have lower risk of having active trachoma (10), reducing fly densities in rural African has also been demonstrated to reduce active trachoma disease (11): This also bases on a poor socio-economic status of the community. Trachoma is said to have been eradicated from the developed world before intervention programs were implemented just as a result of the economic development of the nation (12).

Vitamin A deficiency as manifested by Bitot's spot was found in 14/826 (1.7\%) children. All these cases were under the age of 6 years and were identified in two villages. It was reported that vitamin A deficiency tends to cluster in small regions such as districts, sub districts, villages and even households attributed to shared practices and environments (13). This is significant when compared with the public health significance value of Bitot's spot of more than $0.5 \%$ of children in the community (14). Apart from this clinical finding, such cases were not supported by biochemical determination of serum vitamin A level. Since few years back, a national effort has been made to eradicate poliomyelitis through an extended program for immunization (EPI). Along with this program, vitamin A supplementation has been carried out for under 6 years old children. However, despite this concerted effort, the clinical finding of Bitot's spot of more than $0.5 \%$ is still indicative of its 
public health significance. One would even expect a higher magnitude has it not been tackled with the on going EPI program.

Error of refraction is a major cause of subnormal and low vision in children. Because children of rural community do not attend school, they are not concerned about reduction of vision for their job such as keeping cattle. A similar study done in Nigeria indicated that $59 \%$ of visual loss in those between 5 and 15 years of age (15). In our study, error of refraction was responsible for $62.9 \%$ (39/62) of subnormal vision in children between 6 and 15 years of age. In a study in India, $5.1 \%$ of children in school had a visual acuity of $<6 / 12$ in the better eye (16). Our survey revealed that $6.3 \%$ of children examined had subnormal vision attributable to error of refraction comparable to the Indian report. There are few $(<10)$ optometry technicians in Ethiopia trained overseas. However, the magnitude of error of refraction as a cause of reduced vision is high. Yet, there is no training center for any level of optometry service in the country which is a priority in relation to vision 2020 .

According to WHO definition of blindness (counting fingers $<3$ meters), no child was found to be blind. However, unilateral blindness was found in $8 / 826(1 \%)$ children. Five of these cases $(62.5 \%)$ were due to corneal scar. The contribution of corneal scar to childhood blindness reported ranges between $40 \%$ and $70 \%$ (17) and our finding falls within this range. The finding from a Tanzanian study attributed corneal scar to a combination of measles infection, Vitamin A deficiency and the use of traditional eye medicine (18). The high prevalence of Bitot's spot in the survey may be taken as an indicator of the contribution of vitamin A deficiency to corneal scar in children of this community. Low immunization and health coverage in our country may also indicate the role of measles and traditional eye medicine in corneal scar (4).

The visual acuity of children under 6 years of age was not taken because of the difficulties encountered to do so. We further observed the difficulty to carry VA testing in order children of rural community who were not exposed to modern schooling because of lack of understandings despite the presence of fluent interpreters. In addition, it was easier to examine the macular area than the disc and other parts of the retina, as children tend to look at the light during ophthalmoscopy. Bitot's spot was only assessed clinically but was not evaluated by serum determination of vitamin A levels. However, the result of our study showed that the majority of the causes of ocular morbidity, subnormal and low visions are preventable or treatable. Health education to encourage personal hygiene and environmental sanitation need to be given with improved water supply. In conclusion, this survey has given certain highlights about ocular morbidity in children of rural community. However, further study of large scale is recommended.

\section{Acknowledgment}

This survey was funded by ORBIS International Ethiopia we are grateful to Dr. Wondu Alemayehu, country director of ORBIS International Ethiopia and Dr. Muluken Melese, Deputy Director for their invaluable support.

\section{References}

1. Strategies for the prevention of blindness in national programmes. WHO/PBL 1997 (3), Geneva.

2. National and regional integrated management of childhood illness (IMCI) plan of action 2001-2002, Department of Family Health, Ministry of Health, Addis Ababa, Ethiopia.

3. Health and health related indicators 1999- 2000, Ministry of Heath, Addis Ababa, Ethiopia.

4. Ethiopian Demographic and Health Survey (EDHS), 2000.

5. Abebe B \& Wondu A. prevalence of trachoma and its determinants in Dalocha District, central Ethiopia. Ophthalmic Epidemiol 2001;8(2-3):119-125.

6. Yoseph W \& Samson B. screening for ocular abnormalities and low vision in school children of Butajira town. Ethiop J Health Dev 2002;16(2):165170.

7. Cook JA. Trachoma and the safe strategy. Community Eye Health 1999;32:49.

8. Strategies for the prevention of blindness in national programs. Geneva: World Health Organization, 1984.

9. Lewallen $S$ \& Courtright $P$. Blindness in Africa: present situation and future needs. Br J Ophthalmol 2001;85: 897-903.

10. West SK. Congdon N, Katala S, et al. Facial cleanliness and risk of trachoma in families. Arch Ophthaloml. 1991; 109: 855-7.

11. Emerson PM, Lindsay SW,Walraven GEL,et al. Effect of fly control on trachoma and diarrhoea. Lancet 1999; 353:1401-3.

12. Munoz B \& West SK. Trachoma: The forgotten cause of blindness. Epidemiologic Reviews 1997;19(2):205-217.

13. Sommer A,West SK,et al. Vitamin A deficiency: Health, Survival and Vision. Oxford University press, New York, NY; 1996.

14. Periodic, large oral doses of vitamin A for the prevention of vitamin A deficiency and xerophthalmia: a summary of experiences. A report of the international vitamin A consultative group, 1984.

15. Umeh RE. The causes and profile of visual loss in an onchocerciasis-endemic forest savanna zone in Nigeria. Ophthalmic Epidemiol 1999;6:303-15. 
16. Kalikivayi V, Naduvilath TI, Bansal AK, Dandona L.Vsual impairment in school children in southern India. Ind J Ophthalmol 1997;45:129-34.

17. Foster A. Childhood blindness. Eye. 1988, 2 supplement; S27-S36.
18. Foster A and Sommer A. Corneal ulceration, measles and childhood blindness in Tanzania. Br J Ophthalmol 1987;71:331-42. 

